\title{
Current Abstracts
}

\section{COMPLICATIONS/OUTCOMES}

\section{Richard Lindstrom, Robert J. Toohill, Todd A.} Loehrl, Timothy L. Smith. Management of cerebrospinal fluid rhinorrhea: The Medical College of Wisconsin experience. Laryngoscope 2004;114: 969-974

Introduction: The management of cerebrospinal fluid (CSF) rhinorrhea has evolved in recent years. The purpose of this comprehensive retrospective study is to assess issues related to the management of skull base defects associated with CSF rhinorrhea involving the nose and paranasal sinuses.

Methods: A retrospective review of CSF leak management was conducted. This study included patients with CSF rhinorrhea managed by the Department of Otolaryngology, Medical College of Wisconsin, Milwaukee, WI, from 1992 to 2002. Data collected included site of leak, surgical approach, and any recurrence of leak.

Results: Fifty-seven CSF leaks occurred in 53 patients with CSF rhinorrhea originating from the nose or paranasal sinuses. Twenty-eight of the 53 had iatrogenic injuries resulting in CSF rhinorrhea, 16 had leaks from trauma, and 13 developed spontaneous CSF leaks. Ten patients responded to nonoperative management with bed rest with or without lumbar drain placement. Forty-three patients with 47 leaks underwent surgical repair of CSF rhinorrhea, of which 38 resolved after initial repair. Five of these patients developed recurrent
CSF leaks at the repair site but resolved with subsequent surgery. Of these, two initially presented with spontaneous CSF leaks, one patient had a gunshot wound with massive skull base injury, and two recurred after repair of an iatrogenic injury. Factors associated with failure included lateral sphenoid leaks and elevated body mass index (BMI).

Discussion: Multiple approaches to the management of CSF rhinorrhea can be successful. An endoscopic repair results in resolution of CSF rhinorrhea in the majority of cases. Patients with spontaneous CSF rhinorrhea, elevated BMI, lateral sphenoid leaks, and extensive skull base defects are at increased risk for recurrence. Alternative management options may need to be considered in these cases.

Cem Meco, Gerhard Oberascher. Comprehensive algorithm for skull base dural lesion and cerebrospinal fluid fistula diagnosis. Laryngoscope 2004; 114:991-999

Objectives/Hypothesis: Skull base dural lesions and cerebrospinal fluid (CSF) fistulas are potentially vital conditions whose diagnosis can be challenging. The authors' aim was to compose a comprehensive algorithm that combines the most modern diagnostic tools in easily applicable patterns to indicate a possible dural lesion or CSF fistula.

Study Design: Prospective clinical study.

Methods: The authors collected the data of all patients with suspicion of CSF fistula or dural lesion, or both, between January 1999 and 
December 2002. Beta-trace protein, [beta]2-transferrin, and endoscopic and laboratory sodium fluorescein tests; high-resolution computed tomography; and magnetic resonance cisternography were used according to the symptoms and etiological factors. The results of the diagnostic tools that were used and intraoperative findings (in case of an operative treatment) were reviewed.

Results: From 1999 to 2002, 236 patients were evaluated because of suspicion of dural lesion or CSF fistula, mostly after head trauma. Pattern I of the algorithm was applied for head trauma in dural lesion or CSF leak assessment, pattern II for postoperative CSF leaks, pattern III for evaluation of spontaneous CSF rhinorrhea, and pattern IV for the assessment of recurrent pneumococcal meningitis related to dural lesions without CSF fistula. By applying the patterns of this algorithm, a dural lesion or CSF leak that was also confirmed intraoperatively was detected in 48 patients.

Conclusion: The four patterns of the new diagnostic algorithm described in the present study enable physicians to reliably clarify suspicions of dural lesions and CSF fistulas and aim to help them choose the best possible management. Each pattern uses the optimal combination of CSF tests and radiological imaging to reach a synergistic effect for precisely detecting dural lesions or CSF fistulas. Accordingly, this improves surgical decisionmaking when necessary.

Tarek H. Khrais, Maurizio Falcioni, Abdelkader Taibah, Manoj Agarwal, Mario Sanna. Cerebrospinal fluid leak prevention after translabyrinthine removal of vestibular schwannoma. Laryngoscope 2004;114:1015-1020

Objectives/Hypothesis: The purpose of the report was to present an update on the authors' results for prevention and management of cerebrospinal fluid (CSF) leak after translabyrinthine approach for vestibular schwannoma.

Study Design: Retrospective case review.

Methods: The study was conducted at Gruppo Otologico (Piacenza, Italy), a tertiary referral center for neurotology and skull base surgery. In all, 710 patients underwent translabyrinthine approach for the removal of vestibular schwannoma at that institution between April 1987 and December 2002. The medical records were retrospectively reviewed to identify tumor size, the incidence of postoperative CSF leak, and its treatment.

Results: The overall rate of CSF leak was $1.4 \%$.

Conclusion: The use of proper surgical technique minimizes the risk of CSF leak. Study results show that the continued application of the authors' proposed preventive measures resulted in the maintenance of a low rate of CSF leak. Immediate management of CSF fistulae helps prevent meningitis.

\section{SURGICAL TECHNIQUES}

Carlos Suarez, Jose L. Llorente, Cristina Munoz, Luis A. Garcia, Juan P. Rodrigo. Facial translocation approach in the management of central skull base and infratemporal tumors. Laryngoscope 2004;114:1047-1051

Objectives/Hypothesis: The objectives were to systematize the indications of various types of facial translocation and to present a technical modification to avoid some sequelae of this approach.

Study Design: Retrospective analysis was made of patients treated with different types of facial translocation approach to resect nasopharyngeal, infratemporal, and sinonasal tumors.

Methods: Thirty-nine patients underwent facial translocation approaches for neoplasms originally involving the nasopharynx (27) infratemporal fossa (7), and nasal cavity (5). Of the 21 malignant tumors, 2 were stage T2, 5 were stage T3, and 14 were stage T4. From the entire series of patients, 10 received a unilateral medial translocation, 3 a bilateral medial translocation, 25 a standard facial translocation, and 1 an extended medial facial translocation.

Results: Fifteen patients (38\%) developed some kind of complication, such as wound infection (nine cases) and osteomyelitis and cerebrospinal fluid leak (five cases each). Two patients died as a result of postoperative complications (5.2\%). Overall 5-year survival for malignant tumors was 
43\%. Neither the histological appearance of the tumor nor the $\mathrm{T}$ stage influenced the survival of patients. Survival of patients with intracranial involvement was significantly decreased compared with patients with involvement of other areas $(\mathrm{P}=.0003)$.

Conclusion: The facial translocation approach offers an excellent exposure in tumors with large degree of involvement of the nasopharynx, infratemporal fossa, nasal cavity, and maxillary sinus. More limited osteotomies are indicated in smaller tumors. The use of midfacial degloving minimizes the number of sequelae of these approaches.

Giuseppe Magliulo, Donato Parrotto, Alessandro Stasolla, Mario Marini. Modified translabyrinthine approach and hearing preservation. Laryngoscope 2004;114:1133-1138

Objectives/Hypothesis: In 1991, the translabyrinthine approach was modified by sealing the vestibule with bone wax, which allowed preservation of the hearing function in one patient. The present study aimed specifically at evaluating the effectiveness of the modified translabyrinthine approach in preserving hearing function in a group of patients with vestibular schwannoma that involved the internal auditory canal.

Study Design: Prospective study.

Methods: The series consisted of 12 patients with vestibular schwannoma (average age, $49.7 \mathrm{y}$ ). The schwannoma was smaller than $2 \mathrm{~cm}$ in all patients who had surgery. The patients accepted for the study were required to have preserved hearing function.

Results: None of the patients has shown signs of persistence or tumoral relapse on postoperative magnetic resonance imaging. Immediately after surgery, the entire group had excellent facial functionality. Six patients had maintained their hearing function (four in Class 1 and two in Class 2 according to the Gardner-Robertson scale) at the last follow-up after surgery. A patient during follow-up noted fluctuating hearing contemporarily with a tinnitus in the ear that had maintained its hearing, which was attributable to an endolymphatic hydrops. Three of the six patients with preserved hearing complained of persistent tinnitus. None of the patients had any of the complications or consequences of cerebellopontine surgery.

Conclusion: In our patients, Class 1 or 2 hearing was preserved in $50 \%$ of the patients, with no persistence or tumoral relapse. The follow-up has obviously been short, but the first results are encouraging and deserve to be studied further in a more comprehensive survey.

Allison Teresa Pontius, Yadranko Ducic. Extended orbitozygomatic approach to the skull base to improve access to the cavernous sinus and optic chiasm. Otolaryngol Head Neck Surg 2004;130: 519-525

Background: The orbitozygomatic approach to the skull base has evolved over the past century, with a surge in development during the past 20 years. We describe an extension of this technique involving removal of the most inferior portion of the temporal bone to the level of the internal carotid artery as it enters the carotid canal, to further facilitate exposure of tumors in this region.

Methods and Materials: We performed the extended orbitozygomatic approach in a series of 17 patients with a variety of neoplastic lesions. Our case series was reviewed in a retrospective fashion, and our surgical approach is described. The approach is performed safely and effectively by using the spine of the sphenoid and middle meningeal artery as landmarks and then resecting the temporal bone from the temporal craniotomy site to the carotid canal.

Results: The extended orbitozygomatic approach has been performed at our institution over the past 6 years and has provided significantly improved access to this region of the skull base, facilitating tumor extirpation in our patient population without an increase in complications.

Conclusions: The extended orbitozygomatic approach further improves exposure and facilitates surgical dissection in patients with neoplasms of the cavernous sinus, sellar region, interpeduncular region, and upper clivus. The improved access without an increase in complications supports the inclusion of the extended orbitozygomatic approach in the armamentarium of the skull base surgeon. 
Vassilios I. Vougioukas, Ulrich Hubbe, Vera van Velthoven, Thomas M. Freiman, Alexander Schramm, Uwe Spetzger. Neuronavigation-assisted cranial reconstruction. Neurosurgery 2004;55:162167

Objective: Cranial defects resulting from congenital deformities, ablative resection of osseous tumors, traumatic injury, and destructive infectious lesions are often severe enough to warrant surgical reconstruction. In particular cases, satisfactory cosmetic results may be difficult to achieve because of the extent and location of the lesion.

Methods: We evaluated the role of neuronavigation for reconstruction of large cranial defects with prefabricated titanium and intraoperatively constructed neuronavigation-assisted polymethylmethacrylate implants.

Results: Neuronavigation-assisted cranial reconstruction was performed in 14 patients. Surgical procedure, illustrative cases, postoperative results, and apparent benefits of the technique are presented and discussed. In all patients, excellent cosmetic results were achieved.

Conclusion: In cranial reconstruction, neuronavigation is of value not only for intraoperative determination of resection margins but also for preoperative assessment and planning. The combination of navigation techniques with prefabricated or intra-operatively constructed implants enables achievement of excellent cosmetic results.

\section{DISEASE PROCESSES}

Jannis Constantinidis, Helmut Steinhart, Michael Koch, Michael Buchfelder, Anne Schaenzer, Manfred Weidenbecher, Heinrich Iro. Olfactory neuroblastoma: The University of ErlangenNuremberg experience 1975-2000. Otolaryngol Head Neck Surg 2004;130:567-574

Objective: Olfactory neuroblastoma constitutes a rare and, in clinical terms, biologically variable tumor of the nasal cavity, paranasal sinuses, and the base of the skull and presents a challenge to a modern multidisciplinary therapy. Generally ac- knowledged prognostic factors and a standard therapy fail to exist.

Methods: Between 1975 and 2000 we diagnosed and treated 26 patients with an olfactory neuroblastoma. According to Kadish's classification, 1 patient (4\%) showed stage A, 16 patients (53\%) stage $B$, and 11 cases (43\%) stage C. Hyams grading was established in $81 \%$ of all cases. Fifty-two percent were thus classified as low-grade and 48\% as high-grade tumors. Surgical therapy was performed on 23 patients (88.5\%), surgery being the exclusive form of therapy (monotherapy) in 5 of these patients. Combined therapy was carried out in 18 cases (surgery, radiotherapy, chemotherapy).

Results: Currently, 16 of 26 treated patients (61.5\%) are alive. The disease-specific 10- and 15year survival determined according to KaplanMeier is $76.2 \%$. Fifteen-year survival amounts to $86.7 \%$ for smaller tumors (Kadish A/B) and 63.6\% for advanced tumors (Kadish C). Seven (26.9\%) of the overall group of treated patients developed a recurrence. Salvage therapy was successful in 60\% (3 of 5 patients). Fifteen-year survival following salvage therapy amounts to $60 \%$. Patients with high-grade tumors exhibit a significantly reduced 10-year survival (40\%) compared to patients with low-grade tumors (100\%).

Conclusions: The therapy of olfactory neuroblastoma calls for an interdisciplinary multimodal therapeutic strategy, particularly in the case of advanced tumors. Tumor staging and histopathologic grading according to Hyams are important factors for survival and prognosis. Aggressive salvage therapy can lead to a distinct improvement of long-term survival.

Matthew S. Pogodzinski, Stephen G. Harner, Michael J. Link. Patient choice in treatment of vestibular schwannoma. Otolaryngol Head Neck Surg 2004;130:611-616

Objectives: There are options available to patients newly diagnosed with vestibular schwannoma. Our institution employs stereotactic radiosurgery, microsurgical removal, and watchful waiting. There are no studies in the literature 
examining which of these treatment options $\mathrm{pa}^{-}$ tients are choosing.

Study Design and Setting: Using retrospective chart review from January 2000 through December 2001, we noted several variables and patients' initial treatment choices.

Results: During the 24-month study period, 139 patients were seen at our institution with a new diagnosis of vestibular schwannoma and made a clear initial treatment choice. Of these, 32 (23\%) patients elected watchful waiting; 51 (36\%) underwent stereotactic radiosurgery, and $56(40 \%)$ underwent surgical removal. Surgical excision correlated with younger age and larger tumor size.

Conclusions: Our initial hypothesis, that patients choosing treatment would choose stereotactic radiosurgery more than $50 \%$ of the time, was untrue for the time course studied.

Significance: This is the first study to examine patient choice in treatment of vestibular schwannoma.

C. Macandie, J. A. Crowther. Quality of life in patients with vestibular schwannomas managed conservatively. Clin Otolaryngol 2004;29:215218

Since the era of magnetic resonance imaging (MRI) scanning, vestibular schwannomas are being diagnosed earlier, growth has been shown to be static in up to $70 \%$ of cases and patients have admitted to a reduced quality of life following acoustic neuroma surgery. The aim of this study was to assess the quality of life in patients with vestibular schwannomas managed conservatively. Fifty patients with a vestibular schwannoma were identified who were being managed by interval MRI scanning. Fifty patients attending the general otolaryngology clinic with similar symptoms were prospectively recruited. Each group was assessed using the short form 36 (SF-36) health survey. Both groups were adequately age and sex matched and the SF-36 scores were comparable across all eight health domains. acoustic neuromas.

Aziz Abdel, M. Khaled, Sebastien C. Froelich, Elias Dagnew, Walter Jean, John C. Breneman,
Mario Zuccarello, Harry R. van Loveren, John M. Tew, Jr. Large sphenoid wing meningiomas involving the cavernous sinus: Conservative surgical strategies for better functional outcomes. Neurosurgery 2004;54:1375-1384

Objective: The ability to resect meningiomas that involve the medial and anterior compartments of the cavernous sinus has been refuted. In this retrospective study, we determined the efficacy of total resection of meningiomas that invade the cavernous sinus but are restricted to the lateral compartment.

Methods: We reviewed the charts of 38 consecutive patients with sphenocavernous, clinoidocavernous, and sphenoclinoidocavernous meningiomas who underwent surgical treatment. We assessed early and late cranial nerve morbidity, extent of resection, and long-term outcome (mean, $96 \mathrm{mo}$ ).

Results: In all patients, tumors exceeded $3 \mathrm{~cm}$ diameter. In 22 of 24 patients, total microscopic excision was achieved in tumors that involved only the lateral compartment of the cavernous sinus and touched or partially encased the cavernous internal carotid artery (i.e., modified Hirsch Grades 0 and 1, respectively). In 2 of 24 patients, remaining tumor infiltrated the superior orbital fissure. All $14 \mathrm{pa}^{-}$ tients who had tumors that encased (with or without narrowing) the cavernous segment of the internal carotid artery (Hirsch Grades 2-4) underwent incomplete resection. Among 38 patients, mortality was $0 \%$, late cranial nerve deficits remained in 6 (16\%), and late Karnofsky Performance Scale scores exceeded 90 in 34 patients (90\%). Four patients (10.5\%) developed a recurrence or regrowth. Of 20 patients who were treated with either linear accelerator-based stereotactic radiosurgery or fractionated conformal radiotherapy, 11 had residual tumor and a moderate to high prolifera tive index, 4 had atypical tumors and 1 had angioblastic meningioma after total excision, 2 had regrowth, and 2 had recurrent tumors. In 18 (90\%) of the 20 patients who underwent radiation, tumor size was reduced or controlled.

Conclusion: On the basis of this study and a review of the literature, we demonstrate that 
sphenocavernous, clinoidocavernous, and sphenoclinoidocavernous meningiomas of Hirsch Grades 0 and 1 can be excised from the lateral compartment of the cavernous sinus without postoperative mortality and with acceptable rates of morbidity. Residual tumor in the medial compartment (Hirsch Grades 2-4) may be treated with some form of radiation therapy or observation.

Paul Park, William F. Chandler, Ariel L. Barkan, John J. Orrego, John A. Cowan, Kent A. Griffith, Christina Tsien. The role of radiation therapy after surgical resection of nonfunctional pituitary macroadenomas. Neurosurgery 2004;55:100-107

Objective: Radiotherapy after aggressive surgical resection of nonfunctional macroadenoma (NFA) of the pituitary remains controversial. Historically, immediate postoperative radiotherapy has been recommended to decrease risk of recurrence. With the availability of high-resolution imaging, most neurosurgeons now withhold radiation until recurrence. There is relatively little evidence to support this practice, however. This study reviews postoperative results in a large number of patients with NFA, the majority of whom did not undergo prophylactic radiation.

Methods: Of the 258 patients who underwent surgery from 1979 to 1999 for NFA, medical records were available for 176 . Forty-four patients were treated with immediate postoperative radiotherapy after tumor resection, and the remaining 132 patients were followed up with serial imaging studies and treated with radiotherapy only when a recurrence was documented by follow-up imaging.

Results: Patients in the group that received immediate postoperative radiotherapy at time of initial diagnosis and surgery did not differ significantly with respect to age or sex from those in the group that was observed. Five- and 10-year recurrence rates were 2.3 and $2.3 \%$, respectively, for patients who received immediate postoperative radiotherapy, as compared with 15.2 and 50.5\%, respectively, for patients who were followed up and did not receive radiotherapy unless there was evidence of recurrence or progression. No patient had symptomatic recurrence in the group that was observed if consistent follow-up was performed. Of the 26 patients who received radiotherapy at time of tumor recurrence or progression, 18 had adequate follow-up, and in all cases, the tumors either remained stable or regressed.

Conclusion: Withholding radiotherapy after a high-percentage resection of NFA leads to a higher recurrence rate, but it avoids exposing all patients to the risks of radiation. Deferring radiotherapy for patients with complete or near-complete resection seems to be a safe and prudent approach, as our data suggest that recurrences may be detected early with high-resolution imaging and treated effectively with radiation at time of recurrence. Therefore, immediate postoperative radiotherapy may be eliminated for patients with complete or near complete resection of NFA and who agree to undergo close follow-up for a long period.

Makoto Nakamura, Florian Roser, Sharham Mirzai, Cordula Matthies, Peter Vorkapic, Madjid Samii. Meningiomas of the internal auditory canal. Neurosurgery 2004;55:119-128

Objective: Meningiomas arising primarily within the internal auditory canal (IAC) are notably rare. By far the most common tumors that are encountered in this region are neuromas. We report a series of eight patients with meningiomas of the IAC, analyzing the clinical presentations, surgical management strategies, and clinical outcomes.

Methods: The charts of the patients, including histories and audiograms, imaging studies, surgical records, discharge letters, histological records, and follow-up records, were reviewed.

Results: One thousand eight hundred meningiomas were operated on between 1978 and 2002 at the Neurosurgical Department of Nordstadt Hospital. Among them, there were 421 cerebellopontine angle meningiomas; 7 of these $(1.7 \%$ of cerebellopontine angle meningiomas) were limited to the IAC. One additional patient underwent surgery at the Neurosurgical Department of the International Neuroscience Institute, where a total of 21 cerebellopontine angle meningiomas were 
treated surgically from 2001 to 2003. As a comparison, the incidence of intrameatal vestibular schwannomas during the same period, 1978 to 2002, was 168 of 2400 (7\%). There were five women and three men, and the mean age was 49.3 years (range, 27-59 yr). Most patients had signs and symptoms of vestibulocochlear nerve disturbance at presentation. One patient had sought treatment previously for total hearing loss before surgery. No patient had a facial paresis at presentation. The neuroradiological workup revealed a hom! ogeneously contrast-enhancing tumor on magnetic resonance imaging in all patients with hypointense or isointense signal intensity on T1- and T2-weighted images. Some intrameatal meningiomas showed broad attachment, and some showed a dural tail at the porus. In all patients, the tumor was removed through the lateral suboccipital retrosigmoid approach with drilling of the posterior wall of the
IAC. Total removal was achieved in all cases. Severe infiltration of the facial and vestibulocochlear nerve was encountered in two patients. There was no operative mortality. Hearing was preserved in five of seven patients; one patient was deaf before surgery. Postoperative facial weakness was encountered temporarily in one patient.

Conclusion: Although intrameatal meningiomas are quite rare, they must be considered in the differential diagnosis of intrameatal mass lesions. The clinical symptoms are very similar to those of vestibular schwannomas. A radiological differentiation from vestibular schwannomas is not always possible. Surgical removal of intrameatal meningiomas should aim at wide excision, including involved dura and bone, to prevent recurrences. The variation in the anatomy of the faciocochlear nerve bundle in relation to the tumor has to be kept in mind, and preservation of these structures should be the goal in every case. 